\section{EDUCATION}

Research, Innovation and Solutions on-line ${ }^{(-)}$

\section{Electronic Journal of Research}

in Educational Psychology

PSYCHOLOGY

I+D+i

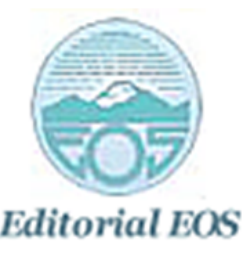

Editorial EOS

\title{
¿Mediante qué estructuras interactivas se relacionan profesorado y alumnado en las aulas universitarias?
}

\section{$\mathbf{M}^{\mathrm{a}}$ del Mar Prados Gallardo ${ }^{1}$, Mercedes Cubero Pérez $^{2}$, Manuel Luis de la Mata Benítez ${ }^{2}$}

${ }^{1}$ Dpto. de Psicología Evolutiva y de la Educación. Universidad de Sevilla ${ }^{2}$ Dpto. de Psicología Experimental, Universidad de Sevilla

\section{España}




\section{Resumen}

Introducción. Los estudiantes universitarios tienen mucho que aportar, y de hecho cuando se les permite así lo hacen, al proceso de construcción conjunta de significados que se da en las aulas.

Método. Se ha utilizado una metodología de corte cualitativo, influenciada por los instrumentos conceptuales y metodológicos provenientes de las aportaciones de determinados aspectos de la Psicología Sociocultural, el Análisis del Discurso, el Análisis Conversacional y la Etnografía Educativa. En este sentido, se ha seguido el desarrollo de una unidad temática en dos aulas universitarias cuyas prácticas pueden calificarse de "buenas prácticas educativas". Se ha analizado la interacción discursiva que se da a lo largo del proceso de enseñanza y aprendizaje desarrollado en las mismas, atendiendo tanto a las formas en que se organiza la actividad conjunta entre profesores y alumnos como a los mecanismos discursivos utilizados en el habla de unos y otros en dicho proceso.

Resultados. Se han identificado determinadas estructuras interactivas que ayudan a que los estudiantes sean protagonistas de sus aprendizajes y el de sus compañeros. Dentro de estas estrategias la conversación se ha mostrado como uno de los medios más efectivos para promover la participación y el aprendizaje en la enseñanza superior.

Discusión y Conclusión. Al igual que la mayoría de propuestas constructivistas sociales encontramos que el aprendizaje puede considerarse como un proceso de "socialización de nuevos modos de discurso" o de adquisición de nuevas formas de comprender y explicar la realidad y que, por tanto, en la enseñanza universitaria, al igual que se ha puesto ampliamente de manifiesto para otros niveles educativos, ha de estar centrada en torno al alumnado, en estructuras interactivas que potencien su protagonismo, y en promover la conversación entre alumnos y con el profesorado.

Palabras Clave: Construcción del conocimiento, enseñanza superior, estructuras interactivas, análisis de la interacción en el aula, análisis del discurso educativo.

Recibido: 16/11/09 Aceptación Inicial: 09/12/09 Aceptación Definitiva: 20/01/10 


\title{
What structures do university teachers and students use in their classroom interaction?
}

\begin{abstract}
Introduction. University students have much to contribute, and in fact they do it when allowed, to the process of meaning construction in the classroom.

Method. In our study we have employed a qualitative methodology, influenced by the conceptual and methodological contributions from Socio-Cultural Psychology, Discourse Analysis, Conversation Analysis and the Ethnography of Education. In this sense, we have followed the development of a thematic unit in two university classrooms characterized as instances of "good practices". Discourse interaction in the teaching-learning process in the classroom was analyzed by attending both to the organization of joint activity between teachers and students and to the discursive mechanisms used in classroom talk.

Results. Some specific interactive structures can help the students to become the protagonists of their learning were identified. Among these strategies, conversation was one of the most effective means/resources to promote participation and learning in higher education.

Discussion and Conclusion. As well as other Social-Constructivist proposals/theoreticians we concluded that learning can be conceived as a process of socialization of new ways of discourse: the acquisition of new forms of understanding and explaining reality and, therefore, university teaching should focus on the students and promote conversation between teachers and students.
\end{abstract}

Keywords: Knowledge construction, higher education, interactive structures, analysis of classroom interaction, educational discourse analysis.

Received: 11/16/09 Initial Acceptance: 12/09/09 Definitive Acceptance: 01/20/10 


\section{Introducción}

Este artículo muestra cómo es posible diseñar y llevar a cabo situaciones de enseñanza y aprendizaje en el ámbito universitario partiendo de una perspectiva constructivista. Desde nuestra perspectiva teórica esto implicaría responder a esta pregunta: ¿de qué forma se relacionan, mediante qué estructuras interactivas, los profesores y alumnos en las aulas universitarias ejemplos de buenas prácticas? De forma más específica nuestros objetivos con este artículo son:

- Proporcionar al profesorado universitario herramientas o estrategias de intervención que faciliten su labor docente como guía de los aprendizajes de sus alumnos.

- Mostrar el papel del alumnado universitario en el proceso de construcción de significados que se da en el aula.

- Acentuar la relevancia de las conversaciones entre alumnos y, entre éstos y el docente, para el proceso de enseñanza y aprendizaje.

Para la consecución de los mismos, en esta introducción teórica se argumentará brevemente, por un lado, la necesidad de una marco teórico sobre el proceso de enseñanza y aprendizaje en los niveles de enseñanza superior que permita interpretar dicho proceso e identificar los elementos favorecedores del buen desarrollo del mismo. Por otro, la relevancia del papel del alumno en su propio proceso de aprendizaje y en el de sus compañeros.

\section{El estudio del proceso de enseñanza y aprendizaje en la universidad}

Tradicionalmente el proceso de enseñanza y aprendizaje ha sido estudiado en los niveles educativos de infantil, primaria y secundaria y, raramente en el ámbito universitario. Así, en el ámbito de la investigación educativa en la universidad aunque los trabajos de investigación existentes son muchos y se encuentran en proliferación continua, sus resultados son controvertidos y predominan los estudios que evalúan la docencia, y no todo el proceso. Se trataría, por tanto, de estudios que parten de la idea de que unas ciertas "variables independientes" del docente (actitudes, aptitudes, estilos de enseñanza,...) son las causantes de los logros o sentimiento de bienestar del alumno o el docente. Además, éstos atienden a aspectos muy variados lo cual dificulta su agrupación, al menos como estrategia didáctica que nos ayuda a comprenderlos. Al mismo tiempo, los resultados que obtienen varían mucho de unas a otras universidades. En el ámbito español, podemos citar algunos trabajos que aportan información 
relevante sobre diversos aspectos como puede ser qué valoran los estudiantes universitarios en sus docentes (Álvarez, 1999; Aparicio, Sanmartín y Tejedor, 1982; Batánaz, 1997), qué características asignan a un buen profesor universitario los propios docentes (García-Valcárcel, 1992; Tejedor, 2003) o la relación existente entre la preferencia de una determinada forma de enseñanza y los enfoque de aprendizaje de los alumnos (Berbén, Pichardo y de la Fuente, 2007). En el ámbito internacional las referencias son aún más y más diversas. Muchos de los trabajos a los que nos podríamos remitir se encuentran recogidos en revistas como "Learning and Instruction" o "Teaching in Higher Education".

Sin embargo, actualmente, al igual que hace ya décadas ocurrió para niveles educativos inferiores, están proliferando investigaciones que examinan las influencias recíprocas de las acciones del profesor y del estudiante. Ejemplos de ello son los trabajos del grupo GRINTIE (Coll, Mauri, y Onrubia, 2005; Coll, Onrubia y Mauri, 2007; Coll, Rochera, Mayordomo y Naranjo 2007; Mauri, Coll, y Onrubia, 2007) acerca de las nuevas tecnologías y la docencia universitaria; los trabajos de Sánchez y sus colaboradores (Sánchez, Rosales, y Cañedo, 1996, 1999) sobre profesores noveles y expertos; los del grupo LAH (Cubero, M., et. al, 2004, Cubero, R. et al., 2003, 2004, 2008; Prados y Cubero, 2007) que estudia los mecanismo discursivos utilizados por profesores y alumnos universitarios en el proceso de construcción de significados, dentro del que podemos enmarcar esta investigación; o los trabajos de Northedge y su grupo (Northege, 2002, 2003a, 2003b) que apoyan la idea de que aprender es hacerse con el discurso de una comunidad y asemejan el proceso de enseñanza y aprendizaje a una excursión por el discurso especializado de una comunidad en la que el profesor actúa como el guía de la misma.

Estos trabajos, como afirman Rosales, Iturra, Sánchez y de Sixte (2006) ${ }^{1}$, toman una noción de "buen aprendizaje" y "buena enseñanza" que es relativamente coincidente, pues se interpreta que se está analizando un proceso que contiene al menos cuatro propiedades: a) debe entenderse desde las reglas de la comunicación humana y, por tanto, con el objetivo de compartir significados; b) tiene una naturaleza colaborativa, en el sentido que las partes (alumnos y profesores) deben tener algún grado de participación activa; c) tiene una naturaleza dinámica, en el sentido de que una de las partes (el alumno) habrá de incrementar su contribución según avanza el proceso, y d) su resultado deseable es una comprensión profunda y 
sustantiva del material. Como se verá más adelante son éstas precisamente las características que definen lo que desde nuestra perspectiva hemos considerado como "buenas prácticas". Esceneraios de actividad en los que se estudiarán las estructurs interactivas en torno a las cuales se relacionan discursivamente profesores y alumnos y a las que se ha hecho referencia en los objetivos.

\section{La efectividad de la interección entre iguales}

Centrándonos en el papel protagonista que un alumno puede tener en su aprendizaje y el de sus compañeros, seguramente todos nos hemos visto alguna vez en la situación de tener que explicar a algún compañero un contenido trabajado en el aula, y al hacerlo, nos hemos vistos forzados a organizar nuestros conocimientos para explicitarlos de una forma clara y comprensible, de manera que nuestra propia comprensión de los mismos ha mejorado. Del mismo modo, nos habremos beneficiado también de las aclaraciones e interpretaciones de otros compañeros pues, como argumentaba Bakhtin (1986), el esfuerzo para comprender la aseveración del otro implica una elaboración y un movimiento activo hacia una respuesta. Con ello, nuestro pensamiento se origina y forma en el proceso de interacción con los pensamientos ajenos (Bakhtin, 1995). Mercer hace referencia a su propia experiencia en situaciones similares y al mismo tiempo afirma que: un buen examen para saber si se comprende bien una cosa es tener que explicárselo a otra persona. $Y$ discutir de manera razonable con alguien al que puedas tratar como a un igual social e intelectualmente es un método excelente para evaluar y revisar tu propia comprensión (Mercer, 1997, p. 99).

En las investigaciones que se han centrado en el estudio de las relaciones que se producen entre iguales trabajando en pequeños grupos encontramos, que a pesar de una falta de explicación integrada sobre cómo la interacción entre iguales puede influir en el desarrollo cognitivo, todas las explicaciones propuestas enfatizan la importancia de los intercambios comunicativos que surgen durante la resolución conjunta de un problema o tarea (Coll y Colomina, 1990; Wood y O’Malley, 1996). Ahora bien, no basta con poner a los alumnos a trabajar juntos para que se produzcan efectos positivos en sus aprendizajes, sino que han de darse una serie de condiciones. Desarrollar los factores y variables que condicionan dicha efectividad supone franquear los límites y objetivos de este capítulo. No obstante, podemos enun-

\footnotetext{
${ }^{1}$ Estos autores se refieren a los trabajos del grupo GRINTIE y el llevado a cabo por su propio grupo (Sánchez, 2001 o Sánchez, Rosales y Suárez, 1999), que son los que nos interesan en este momento. Pero además incluyen las propuestas de Dereck Edwards y Neil Mecer (por 
ciar varios factores que destacan la eficacia del aprendizaje cooperativo frente a las estructuras competitivas o individualistas. Así, las actividades de carácter cooperativo favorecen la aparición de conflictos cognitivos entre los aprendizajes -condición necesaria pero no indispensable para que se produzca un aprendizaje significativo- y, además, proporcionan soporte o apoyo para resolver dichos conflictos. No sólo incrementan el rendimiento de los estudiantes, sino que aumentan la motivación intrínseca y actitudes más positivas hacia el aprendizaje, se corresponden con niveles altos de autoestima y favorecen la percepción positiva y el entendimiento con otros compañeros (Johnson, Johnson, Stanne y Garibaldi, 1990 y Johnson, Maruyama, Nelson y Skon, 1981).

Pero al referirnos a interacción entre iguales estamos aludiendo también a la interacción que se da en el grupo-aula cuando los alumnos y alumnas participan de manera activa en el desarrollo de la clase, discutiendo en gran grupo, ya sea con o sin la intervención del profesor. Así, en el aula es frecuente encontrarnos con discusiones dialogadas, o conversaciones, entre el profesor y los alumnos en las que estos últimos no sólo responden a las preguntas del profesor, cosa que sin duda es importante para el proceso de construcción de significados en el aula, sino que, como bien apunta Candela (1993, 1996, 2005), los alumnos participan de muchas maneras. Los trabajos de esta autora se centran en las intervenciones de los alumnos y en las versiones alternativas que éstos pueden construir sobre el fenómeno natural que se esté estudiando en clase, así como su interés en cómo se construyen versiones legitimadas de la realidad en las aulas. Por nuestra parte, nos gustaría destacar que, en nuestras investigaciones hemos podido confirmar cómo los alumnos, cuando intervienen en el aula dirigiéndose al profesor o al grupo clase generan un tipo de habla que puede llegar a cumplir las mismas funciones que el habla de un docente. Así, hemos encontrado que los alumnos, mediante sus intervenciones discursivas, pueden llegar a hacer uso de las mismas estrategias y mecanismos discursivos que sus docentes, modificando y ajustando sus intervenciones cuando se hace necesario, favoreciendo con ello no sólo sus aprendizajes, sino también los de sus compañeros (Prados, 2005; Prados y Cubero, 2007).

Con este trabajo pretendemos contribuir a la elaboración de un marco teórico del proceso de enseñanza y aprendizaje en el ámbito universitario, ya que si pensamos en la formación que reciben los docentes universitarios para ejercer como tales, vemos que la escasez o ausencia de ésta los hace desconocedores de no sólo términos de una determinada visión de la 
enseñanza y del aprendizaje que, para cualquier profesional de la educación obligatoria resultan familiares, tales como concepciones previas, aprendizaje significativo, construcción del conocimiento, andamiaje, zona de desarrollo próximo, etc., sino de cualquier tipo de estrategias de intervención o herramientas al servicio de su nueva profesión. En la última década, y en gran medida como consecuencia de la implantación del Espacio Europeo de Educación Superior, el interés por la formación de los docentes universitarios de cara a la innovación y mejora educativa en este nivel ha dado lugar a una proliferación de cursos, publicaciones científicas y manuales específicos de didáctica universitaria. Es en esta línea en la que surge nuestro trabajo.

De cara a cubrir los objetivos antes expuestos, se presentaran los resultados de nuestro trabajo que dan respuesta a esta pregunta: ¿de qué forma se relacionan, mediante qué estructuras interactivas, los profesores y alumnos universitarios en dos aulas universitarias ejemplos de buenas prácticas? Podemos definir el concepto de estructura interactiva como aquellas formas de organización de la interacción diádica o grupal entre los agentes, profesor/a y alumnos/as. Éstas reflejan la manera en que se da la interacción discursiva entre profesores y alumnos a lo largo de la unidad temática. Esta unidad en base a la cual giran los resultados que aquí se presentan, es al mismo tiempo resultado del análisis de los datos. Se trata de categorías inductivas, derivadas de las decisiones tomadas en base al propio contexto en el que se producen y a nuestros objetivos de investigación, y por tanto, también parte de nuestros resultados. Es por ello que reservaremos la tipología y caracterización de las mismas en el apartado relativo a los resultados. En definitiva, se espera que las estruturas interactivas que aquí se recogen sirvan de cara a proporcionar al profesorado universitario herramientas o estrategias de intervención que faciliten su labor docente como guía de los aprendizajes de sus alumnos, al mismo tiempo que acentuar la relevancia de las conversaciones entre alumnos y, entre éstos y el docente para el proceso de enseñanza y aprendizaje. Todo ello a partir de dos ejemplos concretos, de buenas prácticas educativas, que demuestran que es posible contar con la participación del alumnado en las aulas universitarias. 


\section{Método}

\section{Participantes}

Este estudio está centrado en el análisis de dos unidades didácticas desarrolladas por dos profesores de la Universidad de Sevilla, en concreto de la Facultad de Ciencias de la Educación, así como los alumnos y alumnas que asisten a sus clases. La selección de estos casos ha venido determinada por la oportunidad para aprender que los mismos nos han brindado (Stake, 1998). Es decir, se han seleccionado aquellos casos que permitiesen aprender el máximo posible sobre el objeto de investigación. En este sentido, Glaser y Strauss (1967) proponen el concepto de muestra teórica, como una de las posibles modalidades de muestreo desde aproximaciones metodológicas cualitativas, para referirse a esta "búsqueda de personas y situaciones que puedan ser especialmente relevantes o fructíferas para los fenómenos que interesan estudiar. Es una forma de recoger datos ricos y sugerentes del modo más puro y con la mínima pérdida de tiempo posible" (p. 224). Las características que definen nuestra muestra por tanto son:

Tabla 1. Características de las unidades de observación

\begin{tabular}{lcc}
\hline & Aula A & Aula B \\
\hline Facultad & Ciencias de la Educación & Ciencias de la Educación \\
Titulación & Lic. de Psicopedagogía & Dipl. Educación Especial \\
Carácter & Optativa, $2^{\mathbf{o}}$ cuatrimestre & Optativa, 1er cuatrimestre \\
Curso & $4^{\mathbf{0}} \mathbf{5}^{\mathbf{0}}$ & $1^{\mathbf{o}}, 2^{\mathbf{0}}$ y $\mathbf{3}^{\mathbf{0}}$ \\
Créditos & 4.5 & 5 \\
Media de alumnos asistentes $_{\mathbf{N}^{\mathbf{0}} \text { sesiones grabadas }}$ & 21 & 47 \\
$\mathbf{N}^{\mathbf{0}}$ horas grabadas/transcritas & 7 & 7 \\
\hline
\end{tabular}

\section{Recogida de la información}

Para la observación etnográfica y el registro de la misma, así como para la recogida de información más detallada se han utilizado tres fuentes diferenciadas: grabaciones de las sesiones en vídeo, toma de notas de campo por parte de un observador en el momento de la grabación y entrevista a cada uno de los docentes. 
Una vez seleccionados los docentes con los que se trabajaría, se procedió de la siguiente manera:

a) Concertamos una cita con cada uno para presentarles nuestro proyecto y pedirles su participación y colaboración en la investigación.

b) Luego acordamos la unidad temática a grabar en cada caso. Es decir, se grabaron situaciones elegidas por ellos mismos y en las que se trataban contenidos con los que se sintieran cómodos.

c) Antes de comenzar a grabar se solicitó el permiso de los alumnos. Para ello les dábamos una breve explicación de nuestros intereses.

d) Una vez finalizado el desarrollo de la unidad didáctica en cada caso, y por tanto finalizado el proceso de grabación, entrevistamos a ambos docentes con el fin de conseguir información contextual suplementaria para completar los análisis de las grabaciones de vídeo y obtener información acerca de sus creencias y propósitos respecto al proceso de enseñanza y aprendizaje que pudiese ayudarnos a entender lo que sucedió a medida que se avanzó en la actividad.

e) Al entrevistar a los docentes, además de recoger información relevante, se ha obtenido el material de planificación o programa de las asignaturas en los que se recogen los objetivos, contenidos y metodología de trabajo y evaluación para cada una de las asignaturas, así como los materiales producidos y utilizados en el transcurso de las sesiones.

\section{Procedimiento}

Antes de adentrarnos en el estudio en cuestión nos gustaría apuntar algunas de las decisiones metodológicas que hemos tomado como consecuencia de la influencia de las aportaciones de determinados aspectos de la Psicología Sociocultural, el Análisis del Discurso, el Análisis Conversacional y la Etnografía Educativa. En primer lugar, entendemos que la actividad a analizar ha de ser observada y registrada en el contexto natural en el que se produce. Además, para comprender dicha práctica a analizar, hemos de partir de la lógica de los propios actores. En este sentido, puede servirnos la recolección de información complementaria a las grabaciones que nos ayuden a contextualizar la actividad educativa. Asimismo entendemos que es importante no analizar las intervenciones discursivas de forma aislada ya que la extracción de una intervención de la cadena comunicativa resultaría ficticia pues entendemos que éstas se encuentran en el discurso a modo de eslabones dependiendo cada uno del anterior y posterior y viceversa. Por tanto, creemos que es necesario transcribir todo el discurso pro- 
ducido en las aulas de principio a fin y trabajar sobre las transcripciones, el material audiovisual y las notas etnográficas simultáneamente. Por último, no podemos olvidar que nos encontramos describiendo y profundizando en la comprensión de un escenario de actividad determinado y con unos participantes y actividades que les son propios y que, por tanto, no podemos pretender generalizar nuestros resultados.

\section{Análisis de los datos}

La sistematización de la información recogida para su análisis ha consistido en el $v a$ ciado de los videos en una plantilla de registro, la digitalización y transcripción íntegra del discurso producido en las aulas y la transcripción íntegra de las entrevistas a los profesores. El análisis ha consistido en la descripción detallada de todas las sesiones identificándose qué, para qué y cómo se hace en cada momento, la búsqueda e identificación de mecanismos discursivos y la identificación de las posibles funciones que tienen éstos en el proceso de construcción de significados.

\section{a) Primer visionado de los videos}

Hemos visto todas las grabaciones y las hemos descrito en una hoja de registro. Todos los vídeos han sido vistos por un mínimo de dos investigadores los cuales han completado la correspondiente plantilla de registro. Esta plantilla de registro tiene varios apartados de identificación y dos grandes columnas en las que hemos reseñado la actividad que se está desarrollando en cada momento y anotaciones y observaciones de una primera aproximación a los datos.

Este primer visionado de las grabaciones sirvió para tomar decisiones relevantes para la investigación. Por un lado, sirvió para tener una idea general del proceso de enseñanza y aprendizaje que se dio en estas aulas y poder realizar una primera descripción. Por otro lado, permitió la identificación de los tipos de actividades que se desarrollaban en el transcurso de las sesiones, dando lugar a un primer esbozo de las dimensiones ¿Qué? ¿Para qué? y ¿Cómo?, que posteriormente han sido utilizadas para describir la actividad desarrollada en cada aula.

\section{b) Transcripción integra de las sesiones}


Los videos han sido digitalizados para su posterior transcripción. Las transcripciones han sido tratadas de acuerdo con un sistema de claves de transcripción que contempla los detalles del discurso, así como la observación de los elementos contextuales. Estas convenciones están basadas en el sistema desarrollado por Jefferson (Atkinson y Heritage, 1984; Sacks, Schegloff y Jefferson, 1974). Puede encontrarse una descripción de estas notaciones en la mayoría de los trabajos sobre el Análisis Conversacional (CA) y el Análisis del Discurso (DA) (Antaki y Widdicombe, 1998; Candela, 1999; Edwards, 1997; Edwards y Potter, 1992; Hutchby y Wooffitt, 1998 y Potter, 1996). Estas convenciones intentan captar la naturaleza del habla como actividad social, y el discurso como acciones situadas, articuladas y coconstruidas en la interacción social (Edwards, 1997; Edwards y Potter, 1992; Potter, 1996). El objetivo es captar las características más significativas de la interacción discursiva. Para indicar la entonación o expresión se utilizan los signos ortográficos comunes en la lengua caste1lana. Éstos se han redefinido para indicar ciertas propiedades del discurso (Ej.: un signo de exclamación "!" hace referencia a una entonación ascendente; las "MAYÚSCULAS" indican la elevación de voz, etc.). El objetivo es captar las características más significativas de la interacción discursiva. El referente para marcar una parte determinada de un texto con ciertas características es ese propio texto y el texto adyacente (elevación de tono o acento marcado, cambios en la velocidad, etc.). De este modo, las transcripciones detalladas nos dan información sobre la forma en que se da el discurso: el mayor o menor énfasis puesto en una o varias palabras, los cambios de tono, las respiraciones, las pausas entre palabras o la fuerza relativa con que se pronuncia una palabra. No entraremos en más detalle acerca de las transcricpicones pues las convenciones utilizadas para elaborar las mismas están al servicio del análisis micro al cual no se hace referencia explícita en este documento.

\section{c) Descripción de las sesiones}

Una vez transcrito el discurso de cada sesión e identificadas la diversidad de actividades se volvió a ver los vídeos junto con las transcripciones para obtener una visión más exhaustiva e identificar posibles patrones en el desarrollo de la organización de la actividad. Para este fin se ha utilizado una plantilla de registro. Ésta consta de cinco columnas. La primera indica el momento de la sesión, en horas y minutos, en el que comienza una actividad. Las columnas segunda, tercera y cuarta hacen referencia a las citadas dimensiones ¿Qué? (actividad), ¿Para qué? (función) y ¿Cómo? (estructura interactiva) de lo que está ocurriendo en el aula en cada momento. Por último, encontramos en la quinta columna una breve descripción de la actividad y comentarios relativos a otras observaciones que pensamos que podrían 
sernos de interés (llamadas de atención, interrupciones por causas ajenas al aula,...). Se ha sintetizado la información obtenida a partir de estas descripciones recogiendo la misma de una forma más sistemática en tablas que han dado lugar a un mapa para cada una de las aulas. Éstos han servido en la búsqueda de patrones de actividad, así como para tener una visión global del proceso seguido en cada una para el trabajo de los contenidos y objetivos propuestos.

Aunque el trabajo de investigación del que se desprenden los resultados que se muestran supone una labor más exhaustiva y a nivel más micro con las transcripciones del discurso educativo de ambas aulas, dentendremos aquí la explicación del proceso seguido en nuestra investigación pues los resultados a los que nos referiremos son los obtenidos a partir del análisis más macro a cuyo procedimiento ya hemos hecho referencia. Concretamente, las estructuras interactivas que hemos identificado en ambas unidades de observación, o la manera en que se da la interacción discursiva entre profesores y alumnos a lo largo de sendas unidades temáticas.

\section{Resultados}

Las estructuras interactivas que se presentan son el resultado de la descripción del desarrollo de la actividad llevada a cabo en ambas aulas. Concretamente hacen referencia a la dimensión ¿Cómo? Se entiende por estructuras interactivas, como ya se ha hecho mención, aquellas formas de organización de la interacción diádica o grupal entre los agentes participantes en un escenario de actividad concreto, en nuestro caso profesor y alumnos. Reflejan la manera en que se da la interacción discursiva entre profesores y alumnos a lo largo de la unidad temática. A continuación se presentan las estructuras interactivas identificadas. En la tabla 2. se presentan las estructuras interactivas que comparten ambas aulas. En las tablas 3. y 4., aquellas estructuras interactivas identificadas en el Aula A y en el Aula B respectivamente. 
Tabla 2. Estructuras interactivas identificadas en ambas aulas

\begin{tabular}{|c|c|c|}
\hline $\begin{array}{l}\text { Estructura } \\
\text { interactiva } \\
\end{array}$ & Representación gráfica ${ }^{2}$ & Definición \\
\hline $\begin{array}{l}\text { Exposición del } \\
\text { profesor (P ante } \\
\text { el grupo aula) }\end{array}$ & $\mathrm{a}$ & $\begin{array}{l}\text { Monólogo del profesor en el que retoma } \\
\text { información dada, presenta información } \\
\text { nueva, conecta ambas o pide una tarea al } \\
\text { alumnado. La posibilidad de intervenir de } \\
\text { los oyentes es limitada o nula. No se consi- } \\
\text { deran las intervenciones puntuales del do- } \\
\text { cente estableciéndose un mínimo de dura- } \\
\text { ción de } 2 \text { minutos para considerar una in- } \\
\text { tervención en esta categoría, a excepción de } \\
\text { las que se da al final de las sesiones pues } \\
\text { suelen ser muy breves. }\end{array}$ \\
\hline $\begin{array}{l}\text { Presentación } \\
\text { dialogada }\end{array}$ & $\mathrm{a}$ & $\begin{array}{l}\text { Momentos en los que el profesor retoma } \\
\text { información dada, presenta información } \\
\text { nueva o conecta información nueva y dada, } \\
\text { o pide una tarea apoyándose en las inter- } \\
\text { venciones de los alumnos que, o bien con- } \\
\text { testan a sus preguntas o bien intervienen } \\
\text { por voluntad propia pues la actividad se } \\
\text { presta a ello. En este tipo de estructura es } \\
\text { fácilmente identificable encadenaciones de } \\
\text { IREs. }\end{array}$ \\
\hline Discusión & & $\begin{array}{l}\text { Se trata de aquellos momentos interactivos } \\
\text { en los que los alumnos y alumnas, princi- } \\
\text { palmente, discuten en torno a una temática } \\
\text { concreta. El discurso predomi-nante es el } \\
\text { del alumnado, aunque el del profesor tam- } \\
\text { bién está presente en menor medida. Éste } \\
\text { hace de moderador de la actividad dando } \\
\text { los turnos y guiando el contenido de la } \\
\text { discusión. Puede parecerse a la presenta- } \\
\text { ción dialogada en cuanto a que a veces se } \\
\text { compone de preguntas y respuestas, pero } \\
\text { existe mayor intercambio entre los partici- } \\
\text { pantes y, las evaluaciones a las respuestas } \\
\text { las dan los propios alumnos más que el } \\
\text { profesor. Estas discusiones terminan bien } \\
\text { con un cambio de actividad, bien con un } \\
\text { cierre de la temática discutida por parte del } \\
\text { profesor. }\end{array}$ \\
\hline No procede & & $\begin{array}{l}\text { El profesor y/o alumnos se encuentran dis- } \\
\text { persos, preparando los materiales o recur- } \\
\text { sos necesarios para realizar alguna de las } \\
\text { actividades. Ej.: encender el retroproyector, } \\
\text { buscar transparencias,... }\end{array}$ \\
\hline
\end{tabular}

\footnotetext{
${ }^{2}$ Hemos utilizado la letra P para representar al profesor y la A para los alumnos. Éstas se encuentran en mayúscula o minúscula en función del protagonismo del interlocutor en cada momento. Las flechas indican el sentido del discurso. Es decir, quién habla y quién escucha.
} 
Tabla 3. Estructuras interactivas identificadas en el aula $\mathrm{A}$

\begin{tabular}{lll}
\hline \multicolumn{1}{c}{$\begin{array}{c}\text { Estructura } \\
\text { interactiva }\end{array}$} \\
$\begin{array}{l}\text { Exposición de un } \\
\text { alumno/a }\end{array}$ \\
$\begin{array}{l}\text { Preguntas y/o } \\
\text { comentarios de } \\
\text { alumnos sin res- } \\
\text { ponder }\end{array}$
\end{tabular}

Tabla 4. Estructuras interactivas identificadas en el aula B

\begin{tabular}{lll}
\hline $\begin{array}{c}\text { Estructura } \\
\text { interactiva }\end{array}$ \\
Pequeños grupos \\
de P)
\end{tabular}

De cara a una mejor comprensión de esta tipología de estructuras interactivas se presentan ejemplos de transcripciones de fragmentos discursivos que han sido categorizados bajo las mismas. En los anexos 1, 2 y 3 se muestran ejemplos de aquellas estructuras interactivas 
que se comparten en ambas aulas: exposición del profesor, presentación dialogada y discusión. El anexo 1 es un ejemplo de una intervención del profesor del aula B que ha sido categorizada como monólogo del profesor. Como puede observarse, aunque el profesor incluye en su discurso preguntas, la posibilidad de intervenir de los alumnos es limitada o nula pues no cede el suficiente tiempo de espera para que éstas sean respondidas. Obsérvese que el profesor inicia su intervención con una afirmación respecto al contenido que están trabajando en ese momento, -la importancia de las ideas previas de los niños en el proceso de enseñanza y aprendizaje- da su opinión, "las ideas previas deben tenerse en cuenta a la hora de la evaluación" y, acto seguido, plantea a los alumnos si lo que ha dicho es correcto o no. Sin embargo, no crea intersticios comunicativos, no hay un silencio o pausa lo suficientemente amplio como para que alguien intervenga. Es él quien responde a la pregunta haciendo así su discurso más dialógico en el que los interlocutores, de algún modo, se encuentran presentes.

En el ejemplo del anexo 2 (Exposición dialogada) el profesor se apoya en las intervenciones de los alumnos para trabajar un determinado contenido. En lugar de presentar la información de manera expositiva, pregunta a sus interlocutores de tal modo que los significados que se tratan en el aula se construyen entre todos. Como se puede observar en este tipo de estructura es fácilmente identificable encadenaciones de IREs. Se presenta en el anexo 3 (Discusión) un ejemplo referido al aula A en el que los alumnos y alumnas, principalmente, discuten en torno a una temática concreta: “¿Es lo mismo una persona experta que una persona estratégica?" Como se puede observar el discurso predominante es el del alumnado, aunque el del profesor también está presente. Se trata de la estructura interactiva en la que más claramente se observa la participación de los alumnos en el proceso de construcción conjunta de significados en el aula.

Se ha elaborado un mapa de estructuras interactivas para cada aula. En éstos se recoge gráficamente la estructura interactiva que se da cada dos minutos en cada una de las sesiones. A partir de estos mapas se ha obtenido información relevante sobre, entre otros factores, el papel que se da a los alumnos en estas aulas. Se han representado las estructuras interactivas antes definidas con distintas tramas lo cual ayuda a ver gráficamente el tiempo en el que los alumnos participan discursivamente en el desarrollo de la unidad y, por tanto aquellos momentos en los que éstos tienen un mayor protagonismo. Estos mapas dan una visión general de las distintas formas en que se relacionan discursivamente los profesores y los alumnos de estas aulas a lo largo de las distintas sesiones que conforman la unidad temática analizada en ambas aulas. Puede diferenciarse así, entre aquéllas estructuras interactivas en las que el dis- 
curso predominante es el del docente (exposiciones del profesor y presentaciones dialogadas, que se dan en ambas aulas) y aquéllas en las que predomina el discurso del alumnado (discusiones en ambas aulas y exposición de un alumno/a en el caso del Aula A y trabajo en pequeños grupos en el caso del Aula B). Atendamos, por un momento, a cada aula por separado.

Centrándonos en el Aula A (ver tabla 5), si tenemos en cuenta que los tonos amarillos hacen referencia a momentos en los que bien un alumno está exponiendo, bien se está dando una discusión a nivel de grupo-aula, podemos afirmar que el protagonismo de los alumnos de este aula es, sin duda, elevado. Como se puede apreciar, la estructura interactiva menos frecuente es la que corresponde a aquellos momentos en que los alumnos dirigen preguntas al compañero que ha expuesto pero no hay feedback por parte de éste, preguntas sin contestar. Seguidamente encontramos las exposiciones o momentos ocupados por monólogos del profesor, en siguiente lugar, las conversaciones entre el profesor y un alumno, presentaciones dialogadas y exposiciones de los alumnos, hasta llegar a las discusiones, que son las que mayor presencia tienen. Hemos destacado en cursiva aquellas estructuras en las que predomina la actividad discursiva de los alumnos.

Conforme se avanza en la unidad (obsérvese la tabla 5), encontramos en las sesiones que el protagonismo y control por parte del profesor y los alumnos va variando. Por ejemplo, el número de discusiones disminuye frente al aumento de conversaciones entre el profesor y el alumno que ha expuesto. Dado que se ha observado etnográficamente el desarrollo de las sesiones y que se ha analizado íntegramente el discurso producido en las mismas de forma detallada, se han identificado varios momentos en el desarrollo de la unidad en los que se hace alusión al modo de proceder en las sesiones y el tiempo disponible para el desarrollo de las mismas. En concreto, y si se nos permite por un momento hacer referencia a parte de los resultados encontrados en el análisis micro, ya en la sesión primera, tras la segunda de las exposiciones, el profesor propone que de ahí en adelante, tras una exposición, se formulen todas las preguntas y comentarios seguidos para ser posteriormente contestados. Aun así no llega a cumplirse. En la sesión 2 el profesor recuerda varias veces el modo de proceder acordado y, finalmente en la sesión 5 se opta por hacer todas exposiciones seguidas dejando un turno de preguntas y comentarios sobre las mismas para el final de la clase. No debe extrañarnos, por tanto, la progresión que se aprecia en las estructuras interactivas que se dan en este aula y, con ello, la disminución que se observa en la participación del alumnado y el aumento del control por parte del profesor, pues, como afirman Onrubia (1992,1993), De Gispert y Onrubia (1997) o Rochera (1999, 2000), el proceso de traspaso de control no es lineal, sino que en la práctica hay momentos intermedios que suponen avances y retrocesos en la cesión o 
asunción del control por parte de los participantes a lo largo del proceso de enseñanza y aprendizaje.

Tabla 5. Mapa de estructuras interactivas a lo largo de las sesiones del Aula A

\begin{tabular}{|c|c|c|c|c|c|c|c|c|c|c|c|c|c|c|}
\hline Tiempo & S1 & & S2 & & S3 & & S4 & & S5 & & S6 & & S7 & \\
\hline & $\begin{array}{l}\text { ¿Para } \\
\text { qué? }\end{array}$ & ¿Cómo? & $\begin{array}{l}\text { ¿Para } \\
\text { qué? }\end{array}$ & ¿Cómo? & $\begin{array}{l}\text { ¿Para } \\
\text { qué? }\end{array}$ & ¿Cómo? & $\begin{array}{l}\text { ¿Para } \\
\text { qué? }\end{array}$ & ¿Cómo? & $\begin{array}{l}\text { ¿Para } \\
\text { qué? }\end{array}$ & ¿Cómo? & $\begin{array}{l}\text { ¿Para } \\
\text { qué? }\end{array}$ & ¿Cómo? & $\begin{array}{l}\text { ¿Para } \\
\text { qué? }\end{array}$ & ¿Cómo? \\
\hline 0.00 & $1 / 2$ & 2 & 0 & 0 & 0 & 0 & 3 & 3 & 2 & 1 & 7 & 4 & 0 & 0 \\
\hline 0.02 & & & 2 & 2 & 3 & 3 & & & 3 & 3 & $3 / 1 / 2$ & 3 & $3 / 1$ & 3 \\
\hline 0.04 & 3 & 3 & $3 / 1$ & 3 & & & & & & & & & & \\
\hline 0.06 & & & & & $4 / 1 / 2$ & 4 & & & & & $3 / 1$ & 3 & 4 & 6 \\
\hline 0.08 & & & & & & & $4 / 5$ & 6 & $5 / 4 / 2$ & 6 & & & $4 / 1$ & 4 \\
\hline 0.10 & 2 & 2 & $4 / 1 / 2$ & 4 & & & & & & & & & & \\
\hline 0.12 & & & & & 3 & 3 & & 1 & & & $4 / 1$ & 6 & & \\
\hline 0.14 & & & & & $4 / 1$ & 4 & & & $3 / 1$ & 3 & 7 & 4 & & \\
\hline 0.16 & & & & & & & & 6 & & & $3 / 1$ & 3 & & \\
\hline 0.18 & & & & & $5 / 2$ & 1 & & 4 & & & & & $3 / 1$ & 3 \\
\hline 0.20 & 3 & 3 & & & & & & & & & & & & \\
\hline 0.22 & & & & & & & & & & & & & & \\
\hline 0.24 & & & & & 4 & 4 & 3 & 3 & & & & & $5 / 4$ & 6 \\
\hline 0.26 & 4 & 4 & & & & & & & & & 5 & 6 & & \\
\hline 0.28 & & & & & & & $4 / 1$ & 4 & 4 & 6 & 3 & 3 & 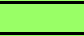 & \\
\hline 0.30 & & & & & & & & & $3 / 1$ & 3 & & & & \\
\hline 0.32 & & & & & & & & & & & & - & 3 & 3 \\
\hline 0.34 & & & $5 / 4 / 1$ & 2 & & & & & 4 & 6 & & & & \\
\hline 0.36 & & & & & & & & & & & $1 / 5 / 4 / 2$ & 4 & 4 & 6 \\
\hline 0.38 & & & & & & & & & 3 & $3 \mathrm{c}$ & 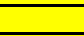 & 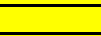 & 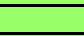 & \\
\hline 0.40 & & & & & & & & & 4 & 6 & & & & \\
\hline 0.42 & $4 / 5$ & 2 & & & & & & & 3 & $3 c$ & $3 / 1$ & 3 & & \\
\hline 0.44 & & & 3 & 3 & & & & & & & $5 / 4$ & 6 & 3 & 3 \\
\hline 0.46 & & & & & $6 / 2$ & 4 & & & & & 7 & 4 & $4 / 1$ & 4 \\
\hline 0.48 & & & & & & & & & 4 & 4 & & & 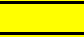 & - \\
\hline 0.50 & & & & & & & & & & & & & & \\
\hline 0.52 & & & 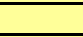 & & 7 & 1 & & 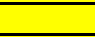 & 7 & 4 & & & - & \\
\hline 0.54 & & & & & & & & & & & & & & \\
\hline 0.56 & & & & & & & 3 & 3 & & & & & & \\
\hline 0.58 & & & $4 / 1 / 2$ & 4 & & & & & & & & & & \\
\hline 1.00 & & & & & & & & & & & & & & \\
\hline 1.02 & & & & & & & 4 & 5 & & & & & & \\
\hline 1.04 & & & & & & & & & & & & & & \\
\hline 1.06 & & & $3 / 1 / 2$ & 2 & & & $4 / 1 / 5$ & 4 & & & & & & \\
\hline 1.08 & & & & & & & & & & & & & & \\
\hline 1.10 & & & & & & & & & & & & & - & \\
\hline 1.12 & & & $3 / 1$ & 3 & & & & & & & & & & \\
\hline 1.14 & & & & & & & & & & & & & 3 & 3 \\
\hline 1.16 & & & & & & & & & & & & & 4 & 6 \\
\hline 1.18 & & & & & & & & & & & & & 3 & 3 \\
\hline 1.20 & & & 4 & 5 & & & & & & & & & -1 & \\
\hline 1.22 & & & & & & & & & & & & & 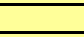 & \\
\hline 1.24 & & & $4 / 1$ & $3 c$ & & & & & & & & & 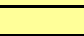 & \\
\hline 1.26 & & & & & & & & & & & & & & \\
\hline 1.28 & & & & & & & & & & & & & 4 & 6 \\
\hline 1.30 & & & & & & & & & & & & & & \\
\hline 1.32 & & & $4 / 1$ & 4 & & & & & & & & & $3 / 1$ & 3 \\
\hline 1.34 & & & & & & & & & & & & & & \\
\hline 1.36 & & & 5 & 2 & & & & & & & & & $5 / 1 / 4$ & 6 \\
\hline 1.38 & & & & & & & & & & & & & & \\
\hline 1.40 & & & & & & & & & & & & & $4 / 1$ & 4 \\
\hline 1.42 & & & & & & & & & & & & & & \\
\hline 1.44 & & & & & & & & & & & & & & \\
\hline 1.46 & & & & & & & & & & & & & & \\
\hline 1.48 & & & & & & & & & & & & & & \\
\hline 1.50 & & & 5 & 1 & & & 7 & 2 & & & & & 7 & 4 \\
\hline 1.52 & & & 4 & 4 & & & & & & & & & & \\
\hline 1.54 & & & & & & & & & & & & & & \\
\hline 1.56 & & & 7 & 1 & & & & & & & & & & \\
\hline 1.58 & & & & & & & & & & & & & & \\
\hline 2.00 & & & & & & & & & & & & & & \\
\hline
\end{tabular}

\begin{tabular}{|c|c|}
\hline Exposición de $\mathrm{P}$ & Discusión \\
\hline Exposición de $\mathrm{P}$ con intervenciones de alumnos & Preguntas sin contestar \\
\hline Exposición de alumno & Conversación entre P y A \\
\hline
\end{tabular}


Tabla 6. Mapa de estructuras interactivas a lo largo de las sesiones del Aula B

\begin{tabular}{|c|c|c|c|c|c|c|c|c|c|c|c|c|c|c|}
\hline \multirow[t]{2}{*}{ Tiempo } & \multicolumn{2}{|c|}{ Sesión 1} & \multicolumn{2}{|c|}{ Sesión 2} & \multicolumn{2}{|c|}{ Sesión 3} & \multicolumn{2}{|c|}{ Sesión 4} & \multicolumn{2}{|c|}{ Sesión 5} & \multicolumn{2}{|c|}{ Sesión 6} & \multicolumn{2}{|c|}{ Sesión 7} \\
\hline & $\begin{array}{l}\text { Para } \\
\text { qué? }\end{array}$ & ¿Cómo? & $\begin{array}{l}\text { Para } \\
\text { qué? }\end{array}$ & ¿Cómo? & $\begin{array}{l}\text { Para } \\
\text { qué? }\end{array}$ & ¿Cómo? & $\begin{array}{l}\text { Para } \\
\text { qué? }\end{array}$ & ¿Cómo? & $\begin{array}{l}\text { Para } \\
\text { qué? }\end{array}$ & ¿Cómo? & $\begin{array}{l}\text { Para } \\
\text { qué? }\end{array}$ & ¿Cómo? & $\begin{array}{l}\text { Para } \\
\text { qué? }\end{array}$ & ¿Cómo? \\
\hline 0.00 & 1 & 1 & 9 & 2 & 1 & 1 & $9 / 2$ & 2 & 2 & 1 & 0 & 0 & 8 & 0 \\
\hline 0.02 & & & & & $8 / 7 / 4$ & 3 & & & & & 9 & 2 & 2 & 1 \\
\hline 0.04 & 6 & 2 & $1 / 2$ & 1 & & & & & 6 & 4 & & & 9 & 3 \\
\hline 0.06 & & & 7 & 3 & & & & & & & & & & \\
\hline 0.08 & 2 & 1 & & & & & & & & & & & & \\
\hline 0.10 & 7 & $3 \mathrm{c}$ & $3 / 5$ & $3 \mathrm{c}$ & & & & & $6 / 3 / 5$ & $3 \mathrm{c}$ & $2 / 8$ & 2 & $3 / 5$ & 1 \\
\hline 0.12 & 8 & 1 & & & & & & & & & & & & \\
\hline 0.14 & 2 & $\overline{1}$ & & & & & & & & & & & 4 & 3 \\
\hline 0.16 & & & & & & & & & & & & & & \\
\hline 0.18 & & & & & & & 0 & 1 & & & & & & \\
\hline 0.20 & 6 & 4 & 8 & 1 & & & 7 & 1 & & & & & & \\
\hline 0.22 & & & $6 / 3 / 5 / 7$ & $3 c$ & & & & & 3 & 2 & 5 & 1 & & \\
\hline 0.24 & & & 8 & 1 & & & 6 & 4 & & & & & & \\
\hline 0.26 & & & $6 / 3 / 5 / 7$ & 3 & & & & & & & & & & \\
\hline 0.28 & & & & & & & & & & & & & & \\
\hline 0.30 & & & 2 & 1 & & & & & & & & & & \\
\hline 0.32 & & & & & & & & & & & & & & \\
\hline 0.34 & & & $3 / 6$ & 5 & & & & & 8 & 1 & & & & \\
\hline 0.36 & $6 / 3$ & 3 & & 2 & & & & & & & & & & \\
\hline 0.38 & & & & 5 & & & & & & & & & & \\
\hline 0.40 & & & & 2 & & & 6 & $3 \mathrm{c}$ & & & & & & \\
\hline 0.42 & & & & & & & & & & & & & & \\
\hline 0.44 & & & & & & & $3 / 5$ & 1 & & & & & & \\
\hline 0.46 & & & & 5 & & & & & & & & & & \\
\hline 0.48 & $3 / 5$ & 2 & & 2 & 8 & 3 & & & & & & & & \\
\hline 0.50 & & & & & $4 / 3$ & $3 \mathrm{c}$ & & & & & 4 & 4 & & \\
\hline 0.52 & 8 & 1 & & & & & $6 / 3$ & 3 & & & & & 0 & 0 \\
\hline 0.54 & & & & & 8 & 2 & 3 & 2 & & & & & & \\
\hline 0.56 & & & & & 9 & $3 c$ & & & & & & & & \\
\hline 0.58 & & & & & & & & & & & $6 / 3$ & 2 & & \\
\hline 1.00 & & & & & & & & & & & & & & \\
\hline 1.02 & & & & & & & & & & & & & $3 / 4$ & 3 \\
\hline 1.04 & & & & & & & & & & & & & & \\
\hline 1.06 & & & 0 & 0 & & & 0 & 0 & & & & & & \\
\hline 1.08 & & & & & & & 9 & 3 & & & & & & \\
\hline 1.10 & & & & & & & & & & & & & & \\
\hline 1.12 & & & 9 & 3 & & & 0 & 0 & & & & & & \\
\hline 1.14 & & & & & & & & & & & & & & \\
\hline 1.16 & & & & & & & & & & & & & & \\
\hline 1.18 & & & $3 / 6$ & 5 & & & $2 / 8$ & 1 & & & & & & \\
\hline 1.20 & & & & 2 & & & 4 & 5 & & & & & & \\
\hline 1.22 & & & $9 / 2$ & 3 & & & & & & & & & & \\
\hline 1.24 & & & & & & & & 2 & & & & & & \\
\hline 1.26 & & & & & & & & 5 & & & 4 & 3 & & \\
\hline 1.28 & & & $6 / 3$ & 5 & & & & 2 & & & & & & \\
\hline 1.30 & & & & & & & & & & & & & & \\
\hline 1.32 & & & & & & & & 5 & & & & & & \\
\hline 1.34 & & & & 2 & & & & & & & & & & \\
\hline 1.36 & & & & & & & & & & & 8 & 1 & & \\
\hline 1.38 & & & & 5 & & & $2 / 8$ & 2 & & & & & & \\
\hline 1.40 & & & & & & & & & & & & & & \\
\hline 1.42 & & & & & & & & & & & & & & \\
\hline 1.44 & & & 8 & 1 & & & & & & & & & & \\
\hline 1.46 & & & & & & & 9 & 1 & & & & & 8 & 1 \\
\hline 1.48 & & & & & & & & & & & & & & \\
\hline 1.50 & & & & & & & & & & & & & & \\
\hline 1.52 & & & & & & & & & & & & & & \\
\hline 1.54 & & & & & & & & & & & & & & \\
\hline 1.56 & & & & & & & & & & & & & & \\
\hline 1.58 & & & & & & & & & & & & & & \\
\hline 2.00 & & & & & & & & & & & & & & \\
\hline
\end{tabular}

\begin{tabular}{|c|c|}
\hline Exposición de P & Discusión \\
\hline Exposición de P con intervenciones de alumnos & Trabajo en pequeños grupos \\
\hline Trabajo en torno a un vídeo & No procede \\
\hline
\end{tabular}

Centrándonos en el Aula B, en el mapa que se recoge en la tabla 6, podemos diferenciar entre aquellas estructuras interactivas en las que el discurso predominante es el del docente (exposiciones del profesor o exposiciones con intervenciones de los alumnos) y aquéllas en 
las que predomina el discurso del alumnado (discusiones). Además, son muchos los momentos dedicados al trabajo en pequeños grupos y en los que, por tanto, los alumnos conversando entre ellos están jugando un papel bastante relevante en el proceso de construcción mutua de significados. Así, si tenemos en cuenta que, al igual que procedimos para el Aula A, hemos utilizado los tonos de amarillo para aquellas estructuras en las que el papel discursivo de los alumnos es más relevante y los tonos celestes para aquéllas en las que es el discurso del profesor el que predomina, vemos que el tiempo que unas y otras ocupan en el desarrollo de la unidad viene a ser semejante. Haciendo referencia a sobre quién recae la responsabilidad y el control a lo largo del desarrollo de la unidad, se puede afirmar que aunque el profesor es quién decide qué y cómo se hace en cada momento, los alumnos juegan un papel bastante activo en el proceso de construcción de significados que se da en este aula. Esta forma en que se distribuye la participación de uno y otros a lo largo de la unidad nos llevan a afirmar que, al igual que ocurría en el caso del Aula A, no se da un traspaso progresivo del control y la responsabilidad por parte del profesor a los alumnos.

Nos gustaría destacar la diferencia que encontramos entre la primera y la última de las sesiones. Así, mientras que en la primera sesión el papel del profesor en el aula parece ser más protagonista que el de los alumnos, en la última de las sesiones, por el contrario, predomina el discurso del alumnado en discusiones en las que se está reflexionando en torno al conocimiento construido a lo largo de la unidad. Este hallazgo resulta coherente pues, por un lado, se sabe, por los trabajos de los autores citados anteriormente, que la existencia de un conjunto amplio de significados compartidos entre el profesor y los alumnos posibilita y favorece la cesión y el traspaso del control y la autonomía del primero a los segundos.

Como se puede apreciar, la estructura interactiva con mayor presencia en este aula es la denominada discusiones, en las que los alumnos tienen un papel bastante relevante con sus intervenciones discursivas. Seguidamente encontramos la presentación dialogada en la que el discurso del profesor es el que predomina, pero hay también intervenciones de los alumnos en las que éste se apoya. Las exposiciones o monólogos del profesor del Aula $\mathrm{B}$, que son más extensas que las del profesor del Aula A, son la siguiente estructura interactiva que predomina. Finalmente, encontramos los pequeños grupos y la estructura referente a cuando trabajan en el aula en torno a un video. Al igual que procedimos con el Aula A hemos destacado en negrita aquellas estructuras en las que predomina la actividad discursiva de los alumnos 
Consideramos que, esta forma de atender a lo que ocurre en un aula, nos aporta información relevante sobre el papel que el docente y los alumnos adoptan en el proceso de construcción de significados. A este efecto, en el siguiente apartado, reflexionaremos sobre estos resultados.

\section{Discusión y Conclusiones}

Centrándonos en el papel de los alumnos en ambas aulas, podemos decir que el tiempo en el que éstos tienen discursivamente un papel activo es muy elevado e incluso mayor que el que tienen los docentes. Así, aunque son éstos quienes dirigen, dan los turnos, deciden y controlan los significados sobre los que se trabaja en el aula, conceden a sus alumnos la oportunidad de conversar y negociar los significados que se construyen entre todos.

En ambas aulas hemos encontrado que se reservan momentos para discutir y otros en los que el docente apoya sus explicaciones en las intervenciones de los alumnos, lo que hemos denominado discusión y exposición dialogadas respectivamente. Además, en el caso del Aula A se pide a los alumnos que ellos mismos expongan un determinado contenido ante sus compañeros y en el caso del Aula B se invita a los alumnos a trabajar en pequeños grupos. Nos hemos referido a estas dos formas de organizar la actividad e interacción que se da en el aula como exposición de un alumno/a y pequeños grupos respectivamente.

Parece que la existencia de estas estructuras interactivas, que fomentan el habla de los alumnos, coincide con la mayoría de las propuestas constructivistas sociales en el sentido de que el aprendizaje escolar puede considerarse como la apropiación por parte de los alumnos y alumnas de las formas de habla y los modos de discurso que son específicos de contextos educativos. Bruner (1988, 1998), Cubero et al. (2008), Edwards (1990), Edwards y Mercer (1988) o Wertsch (1988), por ejemplo, definen el aprendizaje como un proceso de "socialización de nuevos modos de discurso" o dicho de otro modo, de adquisición de nuevas formas de comprender y explicar la realidad. No es que se piense que por el simple hecho de dejar hablar a los alumnos, un profesor parta de concepciones y propuestas constructivistas en su trabajo, o que los alumnos terminen por socializarse con el discurso que se supone que deben adquirir, pero sin duda, es un paso. No sólo porque el que un alumno conozca, discuta y reflexione sobre sus propias ideas sea esencial para el aprendizaje, sino porque se sabe que al trabajar en el aula permitiendo que se produzcan conversaciones entre los alumnos, las ideas 
de unos y otros sobre los diversos contenidos no sólo se explicitan sino que, además, éstas pueden ser tan distintas que entren en conflicto. Incluso es más probable que se dé esta socialización del discurso por parte de los alumnos que en un aula en la que no se les permita o dé la oportunidad de "practicar el discurso de la materia". En este sentido, como se viene afirmando, en ambas aulas se encuentran determinadas estrategias de intervención, que se han traducido a estructuras interactivas, que favorecen el aprendizaje de los alumnos. En las dos aulas son muchos los momentos de discusión, los cuales, se pueden considerar como los más ricos en relación con el proceso de negociación de significados. Pero además, como se ha mencionado, en el aula A se pide a los alumnos que preparen un determinado contenido y lo presenten ante sus compañeros y en el aula B se reservan varios momentos para el trabajo en pequeños grupos. Aunque no se ha analizado a nivel micro los momentos en los que predominan estas estructuras (exposición de un alumno/a y pequeños grupos), se sabe, por la literatura al uso, que a parte de los beneficios que aporta el trabajo entre iguales para la consecución de las metas educativas (Coll y Colomina, 1990; Wood y O’Malley, 1996), explicar a algún compañero o compañera un contenido supone realizar un esfuerzo por organizar los conocimientos propios para explicitarlos de una forma clara y comprensible, de manera que la propia comprensión de quien explica mejora (Mercer, 1997).

Si nos centramos en las tres estructuras interactivas que se comparten en ambas aulas y reflexionamos sobre cada una de ellas, obtenemos una información bastante interesante. Nos referimos a las exposiciones del profesor, exposiciones del profesor con intervenciones de los alumnos o exposiciones dialogadas y discusiones.

Las exposiciones del profesor, o lo que comúnmente suele conocerse como monólogo, han sido poco utilizadas por los dos profesores y cuando lo han hecho era para informar a los alumnos sobre los objetivos del tema, el modo de proceder o el sistema de evaluación de la asignatura (información que normalmente no manejan los alumnos), pedir o comentar una tarea del alumnado o recordar/retomar el conocimiento compartido y enlazarlo con el nuevo. Aunque esto último frecuentemente lo hacen mediante exposiciones dialogadas. Apuntamos esto porque, como afirman Marcelo (2001) y Rosales (2001), la explicación o el monólogo por parte del docente es uno de los discursos más utilizados en las aulas universitarias y, sin embargo, en nuestras unidades de observación no ha ocurrido de esta manera. Ahora bien, esto no quita que pueda haber buenas prácticas cuyo soporte principal sea el monólogo. De hecho, creemos que es posible construir conocimiento a través de exposiciones magistrales 
siempre y cuando en dichas exposiciones puedan identificarse recursos comunicativos y mecanismos semióticos que hagan el discurso más dialógico (Prados y Cubero, 2007), lo cual favorece la posibilidad de que los alumnos receptores puedan establecer coherencia e interrelación entre las ideas presentadas en el discurso expositivo del docente (Cruz de la, 2004; Sánchez, Rosales y Cañedo, 1996; 1999).

En las exposiciones dialogadas, como se expuso al definirlas, son fácilmente identificables encadenaciones de IREs (Iniciación, Respuesta y Evaluación) (Sinclair y Coulthard, 1975). Los profesores de nuestra investigación hacen uso de las mismas principalmente para plantear y resolver dudas en torno al contenido expuesto o destacar un determinando contenido como relevante en el caso del aula A; y para indagar las concepciones de los alumnos y avanzar en el conocimiento que se construye sobre las mismas en el caso del aula B. Esta estructura ayuda a guiar el aprendizaje (Mercer, 1997; Rojas-Drummond y Mercer, 2003) y se encuentran lejos de responder a una estructura en la que el papel del alumno sea pasivo o goce de pocas oportunidades para intervenir, como han puesto de manifiesto autores como Barnes (1976), Lemke (1990) o Stubbs (1979).

En cuanto a las discusiones, éstas han sido definidas como la estructura interactiva relativa a aquellos momentos en los que el discurso predominante es el del alumnado conversando a nivel de grupo aula en torno a una temática concreta. Suelen utilizarse para plantear y resolver dudas, en el caso del aula A, y para explorar las ideas de los alumnos y hacerlos conscientes de ellas e incluso introducir nuevos contenidos sobre la base de las mismas en el caso del aula B. En cualquier caso, se trata de momentos que podemos identificar como de negociación de significados, situaciones de conflictos, desacuerdos o malentendidos en la continuidad del discurso o momentos en los que se explicitan criterios de validación del conocimiento. En este tipo de estructuras el discurso del profesor también está presente, aunque en menor medida. Éste hace de moderador de la actividad dando los turnos y guiando el contenido de la discusión. Puede parecerse a la presentación dialogada en cuanto a que a veces se compone de preguntas y respuestas, pero existe mayor intercambio entre los participantes y las evaluaciones de las respuestas las dan los propios alumnos, más que el profesor. Al igual que Wells y Mejías (2005), hemos encontrado que en los momentos en que hay una discusión, las contribuciones de muchos alumnos no son evaluadas, al menos por el maestro, sino que queda a juicio del resto de compañeros, mediante sus intervenciones, evaluar las mismas. Ahora bien, no se trata de dejar a los alumnos discutir sin más y relegar el papel del docente al 
de moderador imparcial de tal discusión. Por el contrario, en las discusiones de nuestras unidades de observación el profesor no sólo es quien modera las intervenciones, sino que también guía el sentido de los significados que se construyen. Además, si se nos permite aludir, puntualmente, a algunos de los resultados obtenidos en el nivel micro, hemos observado cómo sus intervenciones adquieren mayor peso y relevancia conforme se avanza en el tiempo de una discusión y, frecuentemente, cierra las discusiones, bien legitimando determinados conocimientos o posturas ideológicas de las que se están debatiendo, bien cambiando de actividad. Creemos que esta forma de proceder es parte del éxito de los aprendizajes de los alumnos. Además, sin duda, son los momentos de discusiones los más ricos en relación con el proceso de construcción de significados en ambas aulas. Afirmamos esto ya que en muchas ocasiones, mientras trabajábamos con las transcripciones de las mismas, se han asemejado a las conversaciones exploratorias definidas por Barnes (1976, 2003), Mercer (1996, 1997), Wegerif y Mercer (1997) y Wegerif, Mercer y Dawes (1999). Estos autores se han referido a este tipo de conversación en relación con la calidad de las conversaciones de los niños cuando éstos trabajan juntos y sobre el papel de los profesores para ayudar en estas conversaciones. Se podrían asemejar nuestros dos grupos-aula con uno de los grupos de trabajo analizados por estos autores en los que los interlocutores tratan de forma crítica pero constructiva las ideas de los demás. Así, como se ha obtenido al analizar los mecanismos discursivos encontrados en ambas aulas, resultados que no presentamos en este trabajo, es frecuente la presencia de refutaciones, la petición de aclaraciones y explicaciones, rasgos que caracterizan según los citados autores este tipo de conversación considerada como la más deseable para facilitar el razonamiento.

Quizás gran parte de la bondad de las discusiones que se reconoce en la literatura, referida anteriormente, tenga que ver con el hecho de que este tipo de estructura interactiva favorece especialmente la aparición y resolución del conflicto socio-cognitivo, condición necesaria para que se produzca un aprendizaje significativo. Lo que nos lleva a afirmar con Candela $(1999,2005)$ que cuando a los alumnos, en nuestro caso el alumnado universitario, se les da la oportunidad de intervenir discursivamente tienen mucho que aportar a su propio proceso de aprendizaje.

El estudio de las estructuras interactivas ha sido de utilidad no sólo para la comprensión del proceso de enseñanza y aprendizaje en las aulas universitarias, sino también de cara a la reflexión sobre la posibilidad de cómo podemos influir en la mejora y calidad de la docencia en este ámbito educativo. Creemos, por tanto, que nuestro trabajo da un pequeño paso en 
ese sentido, aportando este tipo de herramientas al servicio del proceso de enseñanza y aprendizaje en la universidad.

\section{Referencias}

Álvarez, V. (1999). Profundizando en la calidad de la enseñanza: aportaciones de los profesores mejor evaluados de la Universidad de Sevilla. Sevilla: Universidad de Sevilla.

Antaki, C. y Widdicombe, S. (1998). Identities In Talk. London: Sage. I

Aparicio, J. J., Sanmartín, R. y Tejedor, F. J. (1982). La enseñanza universitaria vista por los alumnos: Un estudio para la evaluación de los profesores en la enseñanza superior. Madrid: Cuadernos de Capacitación Docente-OEI.

Atkinson, J.M. y Heritage, J. (1984). (Eds.), Structures of social action: studies in conversation analysis. Cambridge: Cambridge University Press.

Bakhtin, M. M. (1979/1995). Estética de la creación verbal. México: Siglo XXI

Bakhtin, M. M. (1986). Speech genres and other late essays. Editado por C. Emerson y M. Holquist. Austin: University of Texas Press.

Barnes, D. (1976). From communication to curriculum. Londres: Penguin.

Barnes, D. (2003). El rol del habla en el aprendizaje. Kikiriki Cooperación Educativa, 68, 2426.

Batanaz, L. (1997). Las variables de relación profesor alumno en el contexto universitario. Revista Electrónica Interuniversitaria de Formación del Profesorado. 1(0) (Recuperado el 4 de agosto de 2009. Disponible en http://www.uva.es/aufop/publica/actas/viii/formafop.htm)

Berbén, B., Pichardo, M.C. y de la Fuente, J. (2007) Relaciones entre preferencias de la enseñanza y enfoques de aprendizaje de los universitarios. Infancia y Aprendizaje, 30(4), 537-550.

Bruner, J. S. (1988). Realidad mental y mundos posibles: los actos de la imaginación que dan sentido a la experiencia. Barcelona: Gedisa.

Bruner, J. S. (1998). Acción, pensamiento y lenguaje. Madrid: Alianza

Candela, A. (1993). La construcción discursiva de la ciencia en el aula. Investigación en la escuela, 21, 31-38.

Candela, A. (1996). La construcción discursiva de contextos argumentativos en la enseñanza de la ciencia. En C. Coll y D. Edwards (Eds.), Enseñanza, aprendizaje y discurso en el 
aula. Aproximaciones al estudio del discurso educacional, (pp. 99-116). Madrid: Fundación Infancia y Aprendizaje.

Candela, A. (1999). Ciencia en el aula. Los alumnos entre la argumentación y el consenso. Mexico: Paidós.

Candela, A. (2005). Students Participation as Co-authoring of School Institucional Practices. Culture and Psycohology, 11(3), 321-337.

Coll, C. y Colomina, R. (1990). Interacción entre alumnos y aprendizaje escolar. En C. Coll, J. Palacios y A. Marchesi (Comps.), Desarrollo psicológico y educación. Vol. II, (pp. 335-352) Psicología de la Educación. Madrid: Alianza.

Coll, C. Mauri, T. y Onrubia, J. (2005). Analyzing actual uses if ICT in formal educational contexts: a sociocultural approach. First ISCAR Crongress. International Society for Cltural and Activity Research. September 20-24, 2005. Sevilla. (Recuperado el 26 de agosto de 2009. Disponible en http://www.ub.edu/grintie)

Coll, C. Onrubia, J. y Mauri, T. (2007). Tecnología y prácticas pedagógicas: las TIC como instrumentos de mediación de la actividad conjunta de profesores y estudiantes. Anuario de Psicología, 38(3), 377-400.

Coll, C., Rochera, M.J., Mayordomo, R. y Naranjo, M. (2007). Evaluación continua y ayuda al aprendizaje. Análisis de una experiencia de innovación en educación superior con apoyo de las TIC. Electronic Journal of Research in Educational Psychology, 13, 5(3), 783-804.

Colomina, R. Onrubia, J. y Rochera, M. J. (2001). Interactividad y mecanismos de influencia educativa y construcción del conocimiento en el aula. En C. Coll, J. Palacios y A. Marchesi (Comps.), Desarrollo psicológico y educación. Vol. II. Psicología de la Educación. (pp. 437-458). Madrid: Alianza.

Cruz Tomé, M.A. de la (2004). Un modelo de lección magistral para un aprendizaje activo y participativo. Cursos y Conferencias de Desarrollo e Innovación Docente. Vigo, 11 y 12 de Noviembre. (recuperado el 25 de enero de 2010 http://webs.uvigo.es/webcalidad/area calidad/documentos/cursos/LeccionMagistralAp untes.pdf)

Cubero, M., García, J., Barragán, A., Cubero, R., De la Mata, M., Santamaría, A., Prados, M.M., y Bascón, M.J. (2004) La construcción del conocimiento psicológico: un estudio de la interacción y el discurso en las aulas universitarias. Las fuentes de validación del conocimiento científico. I Reunión Científica Internacional sobre Etnografía y Educación. Talavera de la Reina, Toledo, España. 
Cubero, R. De la Mata, M. Cubero, M. Santamaría, A. Ignacio, M.J. Prados, M.M. y Ramírez, J.D. (2002). Construction of knowledge on psychology in university classrooms. V Congress of the International Society for Cultural Research and Activity Therory. Ámsterdam.

Cubero, R. de la Mata, M. Cubero, M. Santamaría, A. Prados, M. M. Garcia, J. y Barragán, A. (2003), Knowledge Construction on Psychology in University Classrooms. International Workshop on Cultural- Historical Traditions. Sevilla.

Cubero, R., Cubero, M., De la Mata, M., Santamaría, A., Prados, M. M., Barragán, A., García, J. y Bascón, M.J. (2004a). La construcción del conocimiento psicológico: Un estudio de la interacción y el discurso en las aulas universitarias. La creacción y el mantenimiento de la intersubjetividad. Las fuentes de validación del conocimiento académico. I Reunión Internacional sobre etnografía y educación. Talavera de la Reina, Toledo, España.

Cubero, R., Cubero, M., Santamaría, A., de la Mata, M., Ignacio, M.J. y Prados, M. M. (2008). La educación a través de su discurso. Prácticas educativas y construcción discursiva del conocimiento en el aula. Revista de educación, 346, 71-104.

De Gispert, I. y Onrubia, J. (1997). Analizando la práctica educativa con herramientas socioculturales: traspaso del control y aprendizaje en situaciones de aula. Cultura y Educación, 6/7, 105- 115 .

Edwards, D. (1990) Discourse and the development of understanding in the classroom. En O. Boyd- Barrett y E. Scanlon (Eds.), Computers and Learning. (pp.186-204) Wokinghan: Addison-Wesley.

Edwards, D. (1997). Discourse and cognition. London: Sage.

Edwards, D. y Mercer, N. (1988). El conocimiento compartido. El desarrollo de la comprensión en el aula. Barcelona: Paidós.

Edwards, D. y Potter, J. (1992). Discursive Psychology. London: Sage.

García-Valcárcel, A. (1992). Características del buen profesor universitario según estudiantes y profesores. Revista de Investigación Educativa, 19, 31-50.

Glaser, B. G. y Strauss, A.L. (1967). The discovery of grounded theory: strategies for qualitative research. Nueva York: Aldine.

Hutchby, I. y Wooffitt, R. (1998). Conversation analysis: principles, practices and applications. Cambrigde: Polity Press.

Johnson, D.W., Johnson, R., Stanne, M. y Garibaldi, A. (1990). Impact of group processing on achievement in cooperative groups. The Journal of Psychology, 130(4), 507-516. 
Johnson, D.W., Maruyama, G., Johnson, R.T., Nelson, D. y Skon, L. (1981). Effects of cooperative, competitive and individualistic goal structures on achievement: A metaanalysis. Psychological Bulletin, 84, 47- 62.

Lemke, J.L. (1990). Aprender a hablar ciencia. Lenguaje, aprendizaje y valores. Barcelona: Paidós.

Marcelo, C. (2001). El proyecto docente: una ocasión para aprender. En A. García-Varcárcel, (Coord.) Didáctica Universitaria. (pp.45-57) Madrid: La Muralla.

Mauri, T., Coll, C. y Onrubia, J. (2007) La evaluación de la calidad de los procesos de innovación docente universitaria. Una perspectiva constructivista. Revista de Docencia Universitaria, 1. (Recuperado el 27 de junio de 2009. Disponible en http://www.redu.um.es/Red_U/1/)

Mercer, N. (1996) The quality of talk in children's collaborative activity in the classroom. Learning and Instruction, 6(4), 359- 377

Mercer, N. (1997). La construcción guiada del conocimiento: el habla de profesores y alumnos. Barcelona: Paidós.

Northedge, A. (2002). Organizing excursions into specialist discourse comunities: a sociocultural account of university teaching. En G. Wells y G. Claxton (Eds.), Learning for life in the 21st century: sociocultural perspectives on the future of education (pp. 252264). Oxford: Blackwell Publishers.

Northedge, A. (2003a) Rethinking Teaching in the Context of Diversity. Teaching in Higher Education, 8(1), 17-32.

Northedge, A. (2003b) Enabling participation in academic discourse. Teaching in Higher Education, 8(2), 169-180.

Onrubia, J. (1992). Interacción e influencia educativa: aprendizaje de un procesador de textos. Tesis de Doctorado para la obtención del título de Doctor en Psicología, Facultad de Psicología, Universidad de Barcelona, Barcelona, España.

Onrubia, J. (1993). Interactividad e influencia educativa en la enseñanza /aprendizaje de un procesador de textos: una aproximación teórica y empírica. Anuario de Psicología, 55(3), 83-103.

Prados, M.M. (2005). Construcción del conocimiento y discurso educativo. Una aproximación al estudio de los mecanismos semióticos y estrategias discursivas utilizados por profesores y alumnos en la universidad. Proyecto para la obtención del Diploma de Estudios Avanzados. Departamento de Psicología Evolutiva y de la Educación. Universidad de Sevilla. 
Prados, M.M., y Cubero, R. (2007) Un Acercamiento a la Construcción del Conocimiento en las Aulas Universitarias a Partir del Análisis del Discurso de Profesores y Alumnos. Investigación en la Escuela. 62, 47-61.

Potter, J. (1996). Representing reality. Discourse, rhetoric and social construction. London: SAGE.

Pozo, J. I. (1996). Aprendices y maestros: la nueva cultura del aprendizaje. Madrid: Alianza.

Rosales, C. (2001) Comunicación didáctica en la Universidad. En A. García-Varcárcel, (Coord.) Didáctica Universitaria. (pp.113-153) Madrid: La Muralla.

Rochera, M.J. (1999). Interactividad e influencia educativa: Análisis de algunas actividades de enseñanza y aprendizaje de los primeros números de la serie natural de educación infantil. Tesis de Doctorado para la obtención del título de Doctor en Psicología, Facultad de Psicología, Universidad de Barcelona, Barcelona, España.

Rochera, M.J. (2000). Interacción y andamiaje en el aula: el papel de los errores en la influencia educativa. Cultura y Educación, 17/18, 63- 81.

Rojas-Drummond, S.; y Mercer, N. (2003). Scaffolding the development of effective collaboration and learning. International Journal of Educational Research, 39, 99-111.

Rosales, J., Iturra, C., Sánchez, E. y De Sixte, R. (2006). El análisis de la práctica educativa. Un estudio de la interacción profesor-alumnos a partir de dos sistemas de análisis diferentes. Infancia y Aprendizaje, 2006, 29(1), 65-90.

Sacks, H.; Schegloff, E. and Jefferson, G. (1974). A simplest systematics for the organization of turn-taking for conversation. Language, 50, 696-735.

Sánchez, E. Rosales, J. y Cañedo, I. (1996). La formación del profesorado en habilidades discursivas: ¿Es posible enseñar a explicar manteniendo una conversación encubierta? Infancia y Aprendizaje, 74, 119-137.

Sánchez, E. Rosales, J. y Cañedo, I. (1999). Understanding and Communication in Expositive Discourse: An Analysis of Strategies Used by Expert and Preservice Teachers. Teaching and Teacher Education, 15, 37-58.

Sinclair, J. M. y Coulthard, M. (1975). Towards an analisys of discourse: the English used by teachers and pupils. Oxford: Oxford University Press.

Stake, R. (1998). Investigación con estudios de casos. Madrid: Morata.

Stubbs, M. (1979). Language, Schools and clasrooms. London: Methuen \& Co. Ltd.

Tejedor, F. J. (2003). Un modelo de evaluación del profesorado universitario. Revista de investigación educativa, 21(1), 153-182.

Wegerif, R. \& Mercer, N (1997). A dialogical framework for researching peer talk. En R. 
Wegerif y P. Scrimshaw. Computers and talk in the primary classroom. (pp.48-61) UK: Multilingual Matters Ltd.

Wegerif, R., Mercer, N. y Dawes, L. (1999). From social interaction to individual reasoning: an empirical investigation of a possible socio-cultural model of cognitive development. Learning and Instruction, 9(6), 493-516.

Wells, G. y Mejías, A. (2005). Hacia el diálogo en el salón de clases: enseñanza y aprendizaje por medio de la indagación. Sinéctica, 26, 1-19.

Wertsch, J. (1988). Vygotsky y la formación social de la mente. Barcelona: Paidós.

Wood, D. y O'Malley, C. (1996). Collaborative Learning between Peers. Educational Psychology in Practice, 11(4), 4- 9. 


\title{
Anexos
}

\section{Anexo 1. Ejemplo de monólogo o exposición ${ }^{3}$, del profesor}

\section{B. 67}

\begin{abstract}
67. P: Todo esto tiene que ir asociado al tema de la evaluación. (.) Porque (.) si estamos teniendo en cuenta las ideas que los niños tienen (.) para: e:h utilizarlas didácticamente en nuestras clases, >también habrá que tenerlas en cuenta a la hora de evaluar.< ¿O no? ¿O la evaluación va aparte? (.) Este es un tema que tendremos que discutir posteriormente. Ya se han planteao aquí como dos posturas (.) que habrá que ver e:h la clase se tendrá que decidir (.) porque tenéis que llegar (.) >si es posible, < a un compromiso didáctico. ¿No? Que o (.) >entre toda la gente de la clase ver lo que os parece más apropiado.< <Intentar que los niños vayan subiendo gradualmente estas escaleras, (.) $\underline{y}$ valorar el esfuerzo,> lo que hayan avanzado en la escalera, que es más o menos entiendo yo que es lo que estaba diciendo Lola, ¿no? Era eso ¿no? Valorar ese esfuerzo. ㅇ (.) el otro planteamiento >que es el tradicional y el más extendido en educación que es< yo pongo un tope, un umbral, y digo "los niños tienen que llegar al escalón cuatro y los que no lleguen (.) los cateo. (.) Ese es el planteamiento más tradicional de la escuela, <independientemente del esfuerzo que haya e: realizao cada niño a la hora que subir esa escalera.> Este es un planteamiento que tendremos que discutir, y tendremos que llegar en la unidad siguiente a un acuerdo. Un compromiso didáctico, si nos parece más oportuno, que los niños avancen, y de alguna manera evaluar en función de ese avance o poner un umbral, un tope, y el que no llegue pobrecito él es...
\end{abstract}

\section{Anexo 2. Ejemplo de presentación dialogada}
1B. $11-22$

11. P: Los niños. Eso es. Tenemos que ver qué saben los niños y las niñas sobre el tema de las basuras. Digo que esto ya es más complicao por (.) una cosa que continuamente está saliendo a lo largo del desarrollo de la asignatura y es que las cosas que están en en mi cabeza (.) no tienen por qué coincidir con las cosas que están por ejemplo en la cabeza de la compañera que está aquí comentándole a la otra compañera algo. ¿Lo que tú tienes en tu cabeza es igual que lo que está en mi cabeza?

12. A?: ${ }^{\circ} \mathrm{NO}^{\circ}$

13. P: No ¿Por qué? ¿Por qué no tenemos las mismas ideas?

14. A?: $[---]$

15. P: ${ }^{\circ}$ Espera ${ }^{\circ}$ Vamos a estar en silencio porque si no ella no quiere coger

\footnotetext{
${ }^{3}$ La intervención continúa pero por razones obvias de espacio no mostramos la transcripción del turno completo.
} 


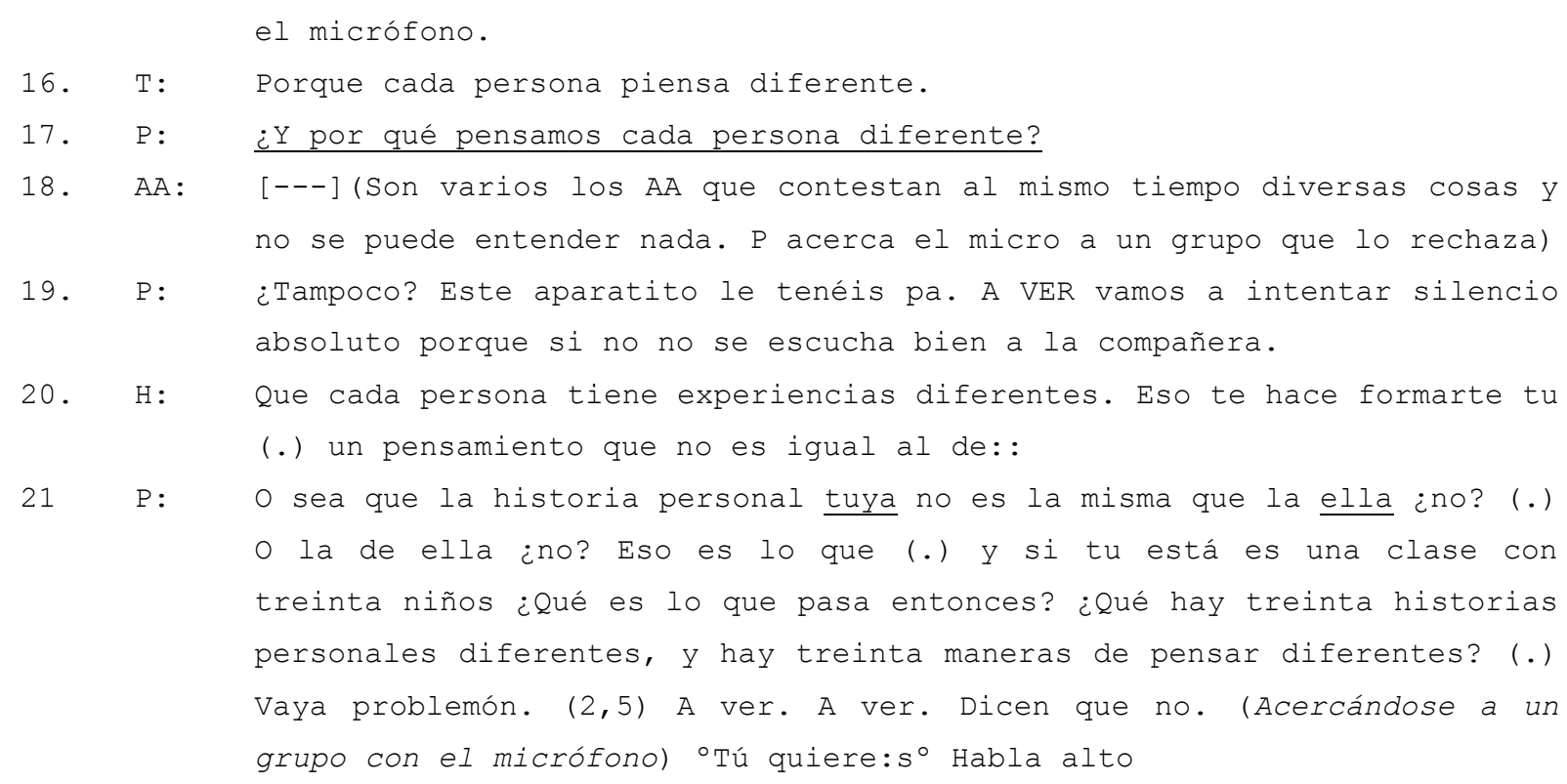

\section{Anexo 3. Ejemplo de Discusión}

\section{A. $186-193$}

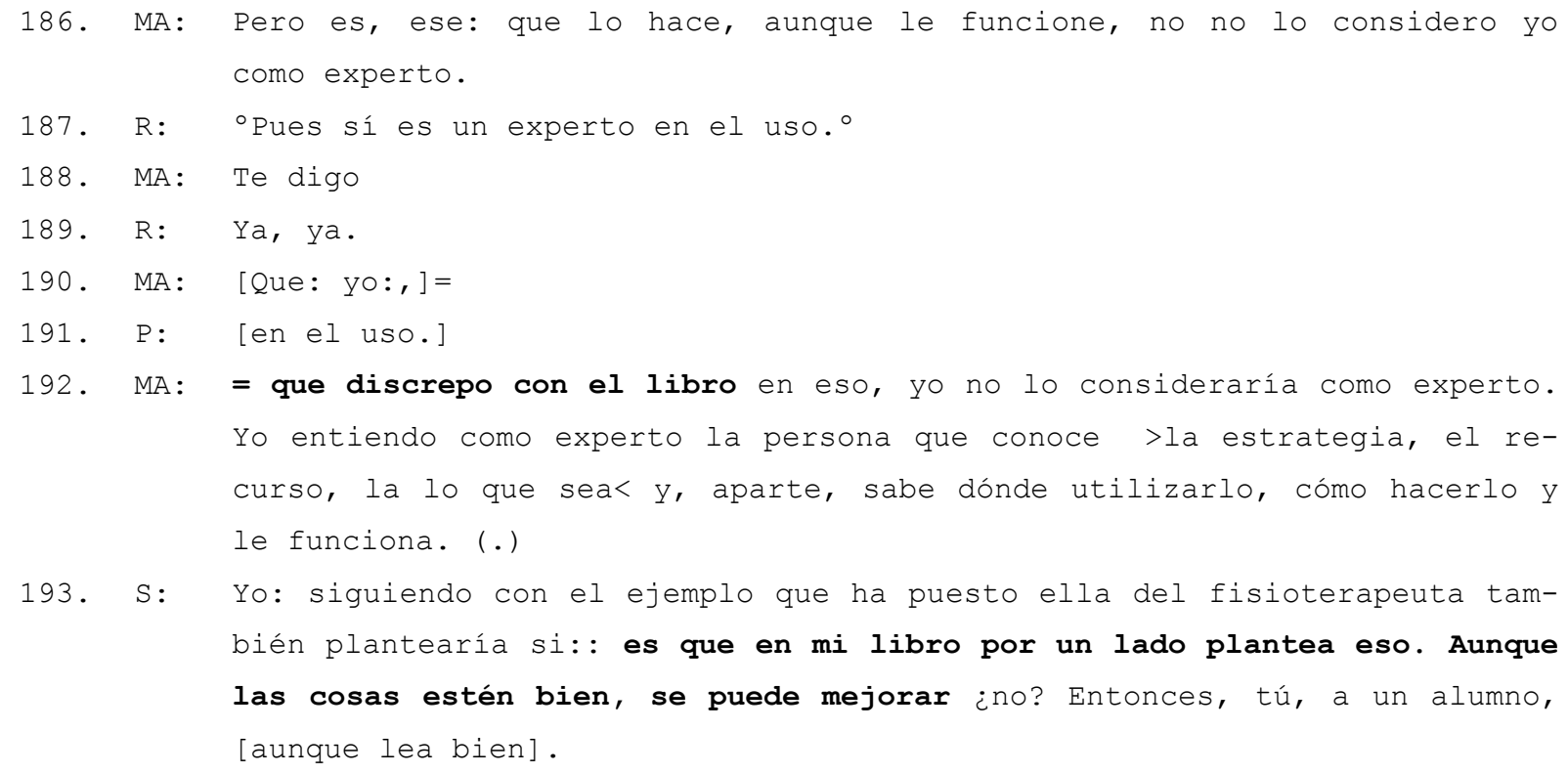

193. S: Yo: siguiendo con el ejemplo que ha puesto ella del fisioterapeuta también plantearía si:: es que en mi libro por un lado plantea eso. Aunque las cosas estén bien, se puede mejorar ¿no? Entonces, tú, a un alumno, [aunque lea bien]. 